\title{
Hepatic Angiomyolipoma with Minimal Intratumoral Fat Content
}

\author{
Masataka Banshodani ${ }^{a} \quad K$ Kohei Ishiyama ${ }^{a}$ \\ Hironobu Amano ${ }^{a}$ Hirotaka Tashiro $^{a}$ Koji Arihiro ${ }^{b}$ \\ Toshiyuki Itamoto ${ }^{a}$ Hideki Ohdan $^{\text {a }}$ \\ aDepartment of Surgery, Division of Frontier Medical Science, Programs for \\ Biomedical Research, Graduate School of Biomedical Sciences, and bepartment \\ of Anatomical Pathology, Hiroshima University, Hiroshima, Japan
}

\section{Key Words}

Angiomyolipoma $\cdot$ Liver tumor $\cdot$ Homatropine methylbromide-45

\begin{abstract}
We report a rare case of hepatic angiomyolipoma with minimal fat content. The low fat content led to an incorrect preoperative diagnosis. A 38-year-old man who was a carrier of hepatitis $B$ virus infection incidentally presented with a hepatic tumor. His serum alpha-fetoprotein level was normal. Ultrasonography revealed a well-circumscribed, heterogeneous hypoechoic nonencapsulated liver tumor measuring $34 \times 24 \mathrm{~mm}$. Precontrast computed tomography (CT) did not reveal fatty attenuation in the lesion. Contrast-enhanced CT revealed a hypervascular nonencapsulated tumor in the arterial phase and moderate washing out of the contrast medium in the portal phase. A hypervascular tumor was observed on CT hepatic arteriography, and complete washing out of the contrast medium on CT during arterial portography. These findings are compatible with hepatocellular carcinoma. The tumor exhibited low signal intensity on T1-weighted images and high signal intensity on T2-weighted images; no hypointensity was observed on fat suppression images. The patient underwent left hemihepatectomy because of a preoperative diagnosis of hepatocellular carcinoma. The histopathological diagnosis was a hepatic angiomyolipoma with $5 \%$ fat content. Low fat content makes the diagnosis of this condition difficult. The absence of serum tumor markers and the presence of a nonencapsulated hypervascular tumor may facilitate the accurate preoperative diagnosis of hepatic angiomyolipomas that have a low fat content and mimic hepatocellular carcinoma.
\end{abstract}




\section{Introduction}

Angiomyolipomas (AMLs) are benign mesenchymal tumors that usually involve the kidney and rarely the liver. Hepatic AMLs are composed of varying portions of fat, epithelioid and spindled smooth muscle cells, and thick-walled blood vessels; these tumors were first described in 1976 by Ishak [1]. The liver is the second most common site of AMLs; hepatic AMLs show an obvious female predominance and occur in patients in widely varying age groups. Patients with hepatic AMLs are usually asymptomatic; the tumor is often found incidentally during health check-ups or medical examinations for other diseases. Patients with large AMLs show symptoms caused by tumor compression $[2,3]$.

Recent advances in imaging modalities and a better understanding of hepatic AMLs have led to an increase in the reported number of cases of this disease. To date, approximately 200 hepatic AMLs have been reported [4]. The imaging features of hepatic AMLs depend on the relative proportions of the tumor components [2]. Accurate preoperative diagnosis of hepatic AMLs is difficult because of their rarity and variable imaging features. It is extremely important to distinguish hepatic AMLs from hepatocellular carcinomas (HCCs) in regions that are endemic for HCC. In most cases, the fat content of hepatic AMLs produces a characteristic appearance on imaging studies, thereby enabling the preoperative differentiation of hepatic AMLs from HCCs [5]. Pathologic diagnosis is made by identification of the three components of AMLs and by positive staining with homatropine methylbromide-45 (HMB-45) [6]. Hepatic AMLs are benign tumors and often grow slowly without producing any clinical symptoms. Recently, however, extremely rare cases of hepatic AMLs with spontaneous rupture, tumor recurrence, and vascular invasion were reported [3].

We report a rare case of asymptomatic hepatic AML with minimal intratumoral fat content. The low fat content led to an incorrect preoperative diagnosis.

\section{Case Report}

A 38-year-old Japanese man was admitted to our hospital for further examination of a hepatic tumor that had been found incidentally during examinations for another medical condition. He had no symptoms due to the liver tumor. He tested positive for hepatitis B surface antibody, hepatitis B envelope antibody, and hepatitis B core antibody and negative for hepatitis B surface antigen, hepatitis envelope antigen, and hepatitis $C$ virus antibody. These findings indicate that the patient was a carrier of hepatitis $B$ virus infection. The results of liver function tests were within the normal ranges, and serum tests for tumor markers, such as alpha-fetoprotein, protein induced by vitamin $\mathrm{K}$ absence or antagonist-II (PIVKA-II), carcinoembryonic antigen, and carbohydrate antigen 19-9, were negative.

Abdominal ultrasonography (US) revealed a well-circumscribed, heterogeneous hypoechoic tumor with a hyperechoic central area; the tumor measured $34 \times 24 \mathrm{~mm}$ and was located in segment 4 of the liver (fig. 1a). Precontrast computed tomography (CT) showed a homogenous, slightly low-attenuated, and well-defined nodule (diameter $27 \mathrm{~mm}$ ); no fatty attenuation was visible within the lesion (fig. $1 \mathrm{~b}$ ). On contrast-enhanced CT, a hypervascular tumor was observed in the arterial phase and moderate washing out of the contrast medium in the portal phase (fig. 1c, d). A hypervascular tumor was observed on CT hepatic arteriography and complete washing out of the contrast medium on CT during arterial portography (fig. 1e, f). Magnetic resonance imaging (MRI) revealed a tumor with low signal intensity on T1-weighted images, high signal intensity on T2-weighted images, and no hypointensity on fat

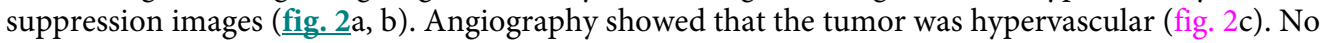
tuberous sclerosis and renal AML were found on physical examination and imaging tests.

The patient underwent left hemihepatectomy because of a preoperative diagnosis of HCC. During surgery, a soft elastic tumor was observed over the left lobe of the liver, which was not cirrhotic. The cut 
surface of the resected specimen was yellowish to tan and round; the tumor was $28 \mathrm{~mm}$ wide and nonencapsulated with clear margins (fig. $3 \mathrm{a}$ ).

On pathological examination, the tumor was found to be composed almost exclusively of epithelioid smooth muscle cells that exhibited a trabecular growth pattern, few thick-walled blood vessels, and few adipose cells (fig. 3b). The fat component accounted for only $5 \%$ of the largest cut surface area of the tumor (fig. 3c). On immunohistochemical analysis, the epithelioid smooth muscle cells were positive for HMB-45 (fig. 3d). On the basis of these findings, the tumor was diagnosed as a hepatic AML. The patient recovered uneventfully and was discharged 8 days after the operation. He is alive and has not shown any evidence of tumor recurrence for 2 years after the operation.

\section{Discussion}

Hepatic AMLs are composed of three components, namely fat, blood vessels, and smooth muscle cells, in varying proportions. Depending on the predominant component, hepatic AMLs are categorized into mixed, lipomatous ( $\geq 70 \% \mathrm{fat})$, myomatous ( $\leq 10 \% \mathrm{fat})$, and angiomatous types. The mixed type of hepatic AML is common [7]. This heterogeneity makes preoperative diagnosis by imaging and needle biopsy difficult. The fat component of AMLs varies from 5 to $90 \%$ of the total volume [8]. The visualization of fat and blood vessels is the most important radiographic feature of AMLs [6]. Thus, the typical imaging features of hepatic AMLs include (a) highly heterogeneous echogenic appearance on US, (b) mixed low density (CT attenuation values $<-20 \mathrm{HU}$ ), (c) high signal intensity on T1- and T2-weighted images, and (d) abundant vessels and tumor staining on angiography [8]. In addition, the radiological demonstration of a nonencapsulated lesion with central vessels may facilitate the preoperative diagnosis [9].

MRI is more sensitive than CT in detecting fat, which appears hypointense on fat suppression images [10]. Therefore, most hepatic AMLs can be conclusively diagnosed using imaging modalities, provided the tumors have a sufficient fat content. Occasionally, it is difficult to distinguish hepatic AMLs from other fat-containing hepatic tumors, such as HCC with fatty metamorphosis, lipoma, and liposarcoma. Hepatic AMLs with low fat content are difficult to diagnose. Tumors that are composed predominantly of myomatous elements are most likely to be incorrectly diagnosed [9]. It is especially difficult to differentiate HCCs from myomatous hepatic AMLs on histology when the latter exhibit trabecular growth patterns [11], as in the case of our patient. Some authors have reported cases of hepatic AMLs with low fat contents and atypical appearances on preoperative imaging tests [12].

On dynamic CT or MRI studies, hepatic AMLs are markedly enhanced in the arterial phase and enhancement persists in the venous phase, depending on the vascularity of the tumor. Such hepatic AMLs should be differentiated from HCCs and cavernous hemangiomas. Ren et al. reported the typical findings of hepatic AMLs: on contrast-enhanced CT, 21 (92\%) of 26 hepatic AMLs were markedly enhanced in the arterial phase, and 18 (69\%) remained enhanced in the venous phase. Prolonged enhancement in the venous phase indicates the proliferation of blood vessels [2]. Ahmadi et al. reported that hepatic AMLs show early and prolonged enhancement $(>4 \mathrm{~min})$ with delayed peak enhancement at $40-80 \mathrm{~s}$ as opposed to HCCs, which show peak enhancement at $10 \mathrm{~s}$ and absent or minimally delayed enhancement [13]. Differences in intratumoral hemodynamics between AMLs and HCCs greatly facilitate the distinction between these two conditions. Yoshimura et al. angiographically determined that hepatic AMLs are drained by the hepatic veins [9]. Further, they suggested that early venous drainage by the hepatic veins can be used to distinguish hepatic AMLs from HCCs, because the latter are drained by the portal veins. 
Despite the existence of several characteristic imaging features of hepatic AMLs, the rate of correct preoperative diagnosis using imaging studies with or without needle biopsy has been reported to be $30-50 \%$, even in recent studies [3]. This low rate of diagnosis can be attributed to the rarity of these tumors and the variations in the proportions of their three components. In this case, we were unable to differentiate a hepatic AML from a typical HCC because of the low fat content of the tumor. Hepatic AMLs are usually not associated with hepatitis virus infections or hepatic dysfunction. It is possible that our patient was a carrier of hepatitis B virus infection. Postcontrast CT scans revealed a heterogeneous enhanced tumor in the arterial phase and washing out of the contrast medium from the tumor in the portal venous phase. These findings are typically observed in HCC [14]. The abdominal US, CT, and MRI findings in this case supported a diagnosis of HCC, even though capsule formation was not detected (approximately $70 \%$ of HCCs with a diameter $>2 \mathrm{~cm}$ are encapsulated) [15].

In conclusion, the low fat content of hepatic AMLs impedes the preoperative imaging diagnosis of these tumors. Owing to the heterogeneity of hepatic AMLs, needle biopsy is not sufficient for preoperative diagnosis. Further, it may pose a risk of peritoneal metastasis if a malignant tumor is present. The normal levels of serum alpha-fetoprotein and PIVKA-II, the visualization of a nonencapsulated and hypervascular tumor and moderate washing out of the contrast medium in the portal phase of contrast-enhanced CT are helpful for the correct preoperative diagnosis of hepatic AMLs that contain little fat and mimic HCC. In such cases, careful observation is recommended for patients with poor hepatic reserve and for elderly patients. However, for other patients, surgical treatment should be considered the first-line treatment if a malignancy cannot be ruled out. 


\begin{tabular}{r|l|l|l} 
Case Reports $/ \mathrm{h}$ & $\begin{array}{l}\text { Case Rep Gastroenterol 2009;3:324-331 } \\
\text { D0I: 10.1159/000255355 }\end{array}$ & Published online: November 20, 2009 & $\begin{array}{l}\text { O 2009 S. Karger AG, Basel } \\
\text { ISSN 1662-0631 } \\
\text { www.karger.com/crg }\end{array}$ \\
\hline
\end{tabular}

Fig. 1. a Abdominal US revealed a well-circumscribed, heterogeneous hypoechoic tumor (arrow) with a central hyperechoic area. The tumor measured $34 \times 24 \mathrm{~mm}$ and was located in segment 4 of the liver. b A precontrast CT scan showed that the lesion was a homogenous well-defined nodule with slightly low attenuation. The tumor had a diameter of $27 \mathrm{~mm}$, and no fatty attenuation was visible within the lesion. c, d Contrast-enhanced CT revealed a hypervascular tumor in the arterial phase (c) and moderate washing out of the contrast medium in the portal phase (d). e, $\mathbf{f}$ A hypervascular tumor was observed on CT hepatic arteriography (e) and complete washing out of the contrast medium was observed on CT arterioportography $(\mathbf{f})$.
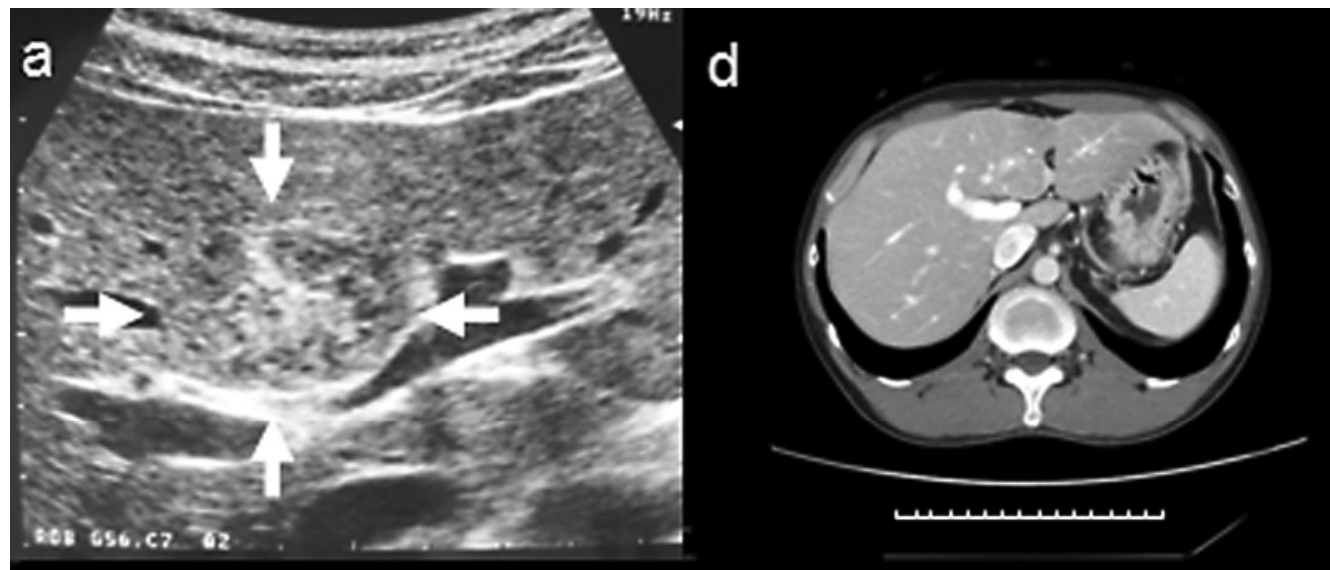

b

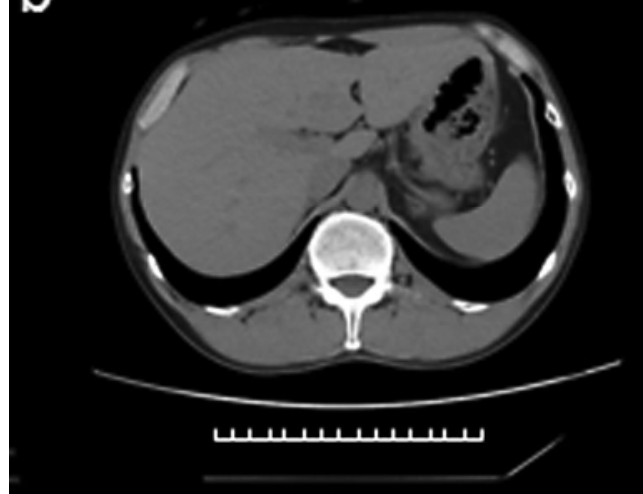

C

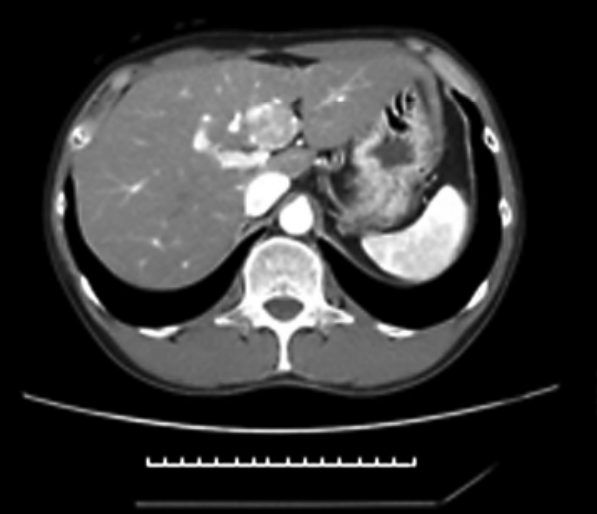

e

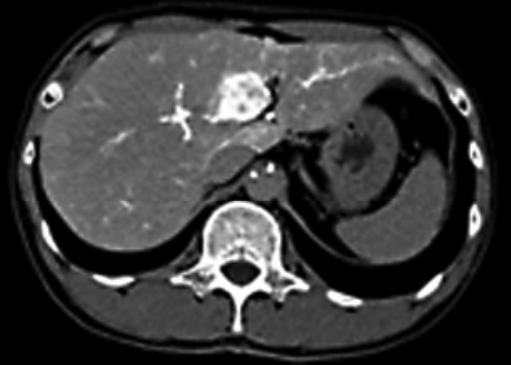

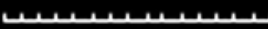

f

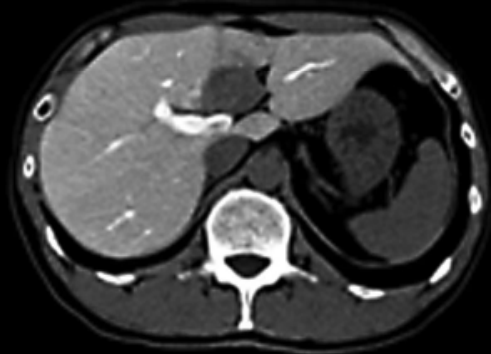

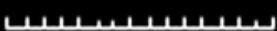




\begin{tabular}{r|l|l|l} 
Case Reports $/ \mathrm{h}$ & $\begin{array}{l}\text { Case Rep Gastroenterol 2009;3:324-331 } \\
\text { D0I: 10.1159/000255355 }\end{array}$ & Published online: November 20, 2009 & $\begin{array}{l}\text { O 2009 S. Karger AG, Basel } \\
\text { ISSN 1662-0631 } \\
\text { www.karger.com/crg }\end{array}$ \\
\hline
\end{tabular}

Fig. 2. a, b MRI revealed a tumor with low signal intensity on T1-weighted images (a) and high signal intensity on T2-weighted images, without any hypointensity on fat suppression images (b). c Angiography revealed an enhanced tumor in segment 4 of the liver. The tumor appeared hypervascular.

\section{a}

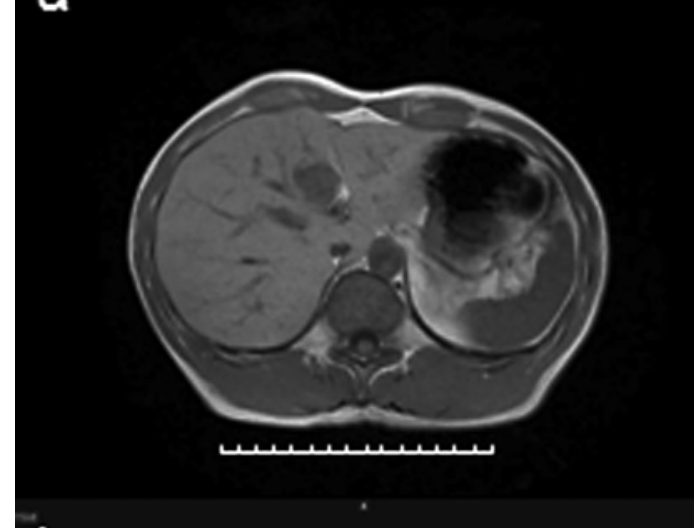

b

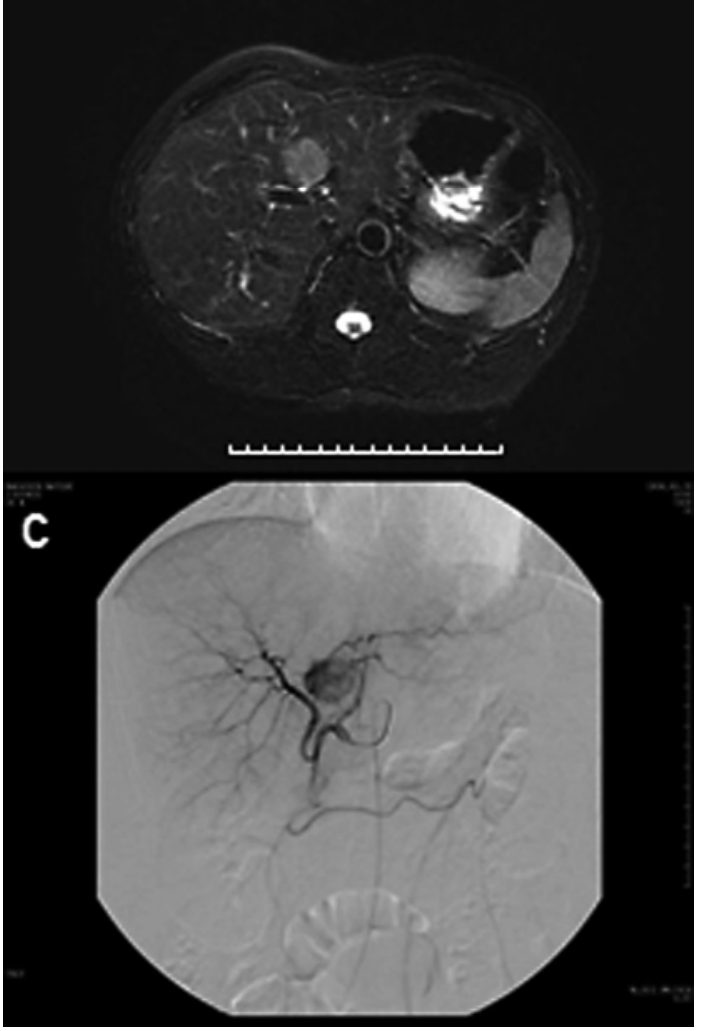




\begin{tabular}{r|l|l|l} 
Case Reports $/ \mathrm{h}$ & $\begin{array}{l}\text { Case Rep Gastroenterol 2009;3:324-331 } \\
\text { D0I: 10.1159/000255355 }\end{array}$ & Published online: November 20, 2009 & $\begin{array}{l}\text { O 2009 S. Karger AG, Basel } \\
\text { ISSN 1662-0631 } \\
\text { www.karger.com/crg }\end{array}$ \\
\hline
\end{tabular}

Fig. 3. a The cut surface of the resected tumor appeared yellowish to tan and round with clear margins. No capsule was seen, and the diameter of the tumor was $28 \mathrm{~mm}$. b, c Hematoxylin and eosin staining of the tumor showed that the tumor was almost exclusively composed of epithelioid smooth muscle cells that exhibited a trabecular growth pattern, few thick-walled blood vessels (arrow), and few adipose cells (b). The fat component accounted for only $5 \%$ of the largest cut surface area of the tumor (c). d On immunohistochemical analysis, the epithelioid smooth muscle cells were positive for HMB-45.

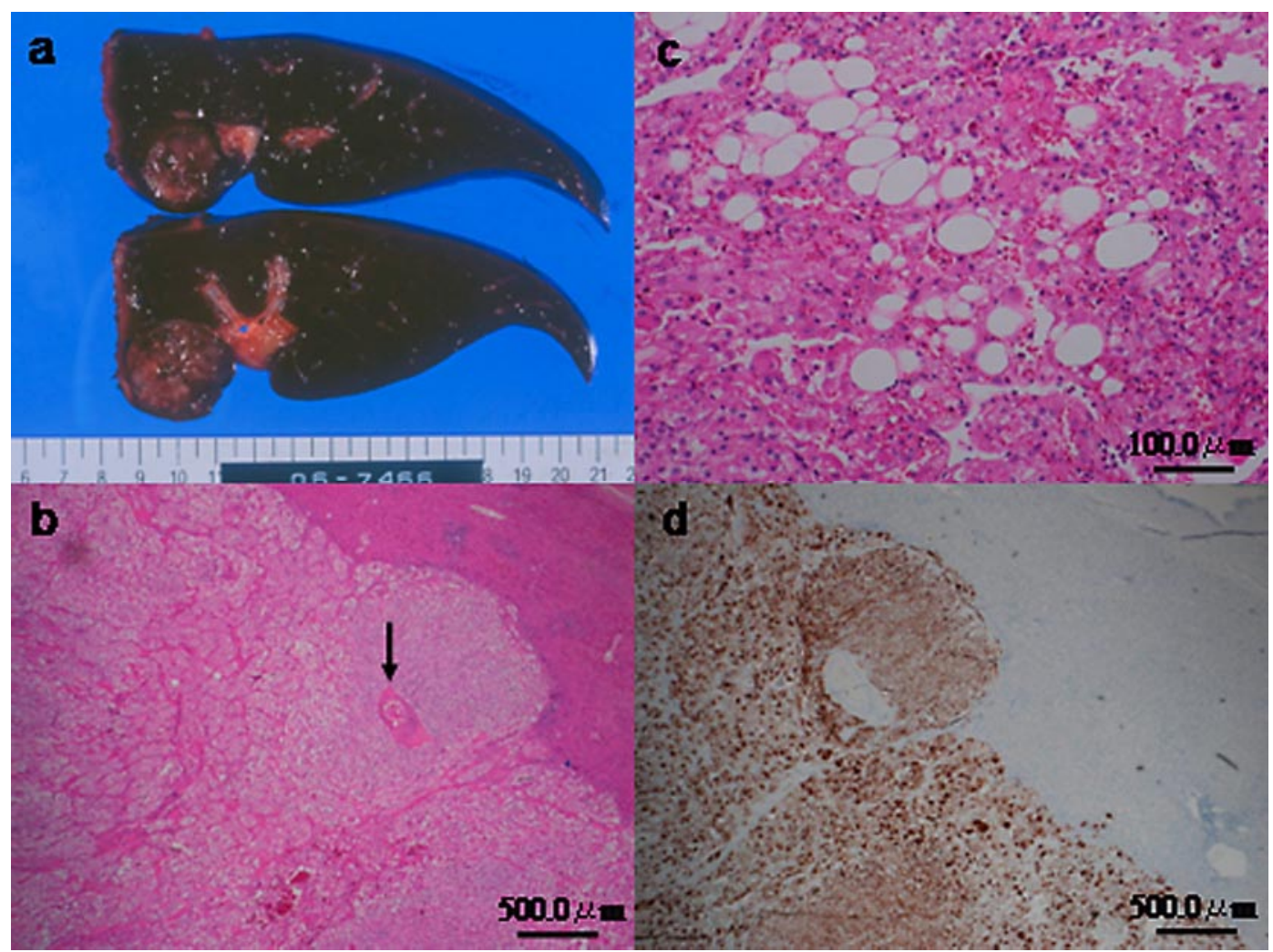




\section{References}

1 Ishak KG: Mesenchymal Tumors of the Liver. New York, John Wiley Medical, 1976, pp 247-307.

2 Ren N, Qin LX, Tang ZY, Wu ZQ, Fan J: Diagnosis and treatment of hepatic angiomyolipoma in 26 cases. World J Gastroenterol 2003;9:1856-1858.

-3 Yang CY, Ho MC, Jeng YM, Hu RH, Wu YM, Lee PH: Management of hepatic angiomyolipoma. J Gastrointest Surg 2007;11:452-457.

-4 Jiang TA, Zhao QY, Chen MY, Wang LJ, Ao JY: Diagnostic analysis of hepatic angiomyolipoma. Hepatobiliary Pancreat Dis Int 2005;4:152-155.

-5 Hogemann D, Flemming P, Kreipe H, Galanski M: Correlation of MRI and CT findings with histopathology in hepatic angiomyolipoma. Eur Radiol 2001;11:1389-1395.

6 Sturtz CL, Dabbs DJ: Angiomyolipomas: the nature and expression of the HMB45 antigen. Mod Pathol 1994;7:842-845.

-7 Tsui WM, Colombari R, Portmann BC, Bonetti F, Thung SN, Ferrell LD, Nakanuma Y, Snover DC, Bioulac-Sage P, Dhillon AP: Hepatic angiomyolipoma: a clinicopathologic study of 30 cases and delineation of unusual morphologic variants. Am J Surg Pathol 1999;23:34-48.

8 Nonomura A, Mizukami Y, Kadoya M: Angiomyolipoma of the liver: a collective review. J Gastroenterol 1994;29:95-105.

-9 Yoshimura H, Murakami T, Kim T, Nakamura H, Hirabuki N, Sakon M, Wakasa $\mathrm{K}$, Inoue Y: Angiomyolipoma of the liver with least amount of fat component: imaging features of CT, MR, and angiography. Abdom Imaging 2002;27:184-187.

10 Hooper LD, Mergo PJ, Ros PR: Multiple hepatorenal angiomyolipomas: diagnosis with fat suppression, gadolinium-enhanced MRI. Abdom Imaging 1994;19:549551.

11 Petrolla AA, Xin W: Hepatic angiomyolipoma. Arch Pathol Lab Med 2008;132:1679-1682.

-12 Terris B, Flejou JF, Picot R, Belghiti J, Henin D: Hepatic angiomyolipoma. A report of four cases with immunohistochemical and DNA-flow cytometric studies. Arch Pathol Lab Med 1996;120:68-72.

13 Ahmadi T, Itai Y, Takahashi M, Onaya H, Kobayashi T, Tanaka YO, Matsuzaki Y, Tanaka N, Okada Y: Angiomyolipoma of the liver: significance of CT and MR dynamic study. Abdom Imaging 1998;23:520-526.

14 Ros PR, Murphy BJ, Buck JL, Olmedilla G, Goodman Z: Encapsulated hepatocellular carcinoma: radiologic findings and pathologic correlation. Gastrointest Radiol 1990;15:233-237.

15 Nakashima T, Kojiro M: Hepatocellular Carcinoma: An Atlas of Its Pathology. Tokyo, Springer, 1987. 